\title{
Journal of Prosthodontic Research
}

\author{
Official Journal of Japan Prosthodontic Society
}

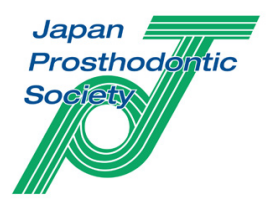

Original article

\section{Bond durability and surface states of titanium, Ti-6Al-4V alloy, and zirconia for implant materials} \author{
Keita Toyoda ${ }^{\mathrm{e}}$, Hiroshi Shimizu ${ }^{\mathrm{c}}$, Hirofumi Kido ${ }^{\mathrm{e}}$ \\ ${ }^{a}$ Center for Oral Diseases, Fukuoka Dental College, Fukuoka, Japan \\ ${ }^{b}$ Section of Removable Prosthodontics, Department of Oral Rehabilitation, Fukuoka Dental College, Fukuoka, Japan \\ ${ }^{c}$ Division of Biomaterials, Department of Oral Functions, Kyushu Dental University, Fukuoka, Japan \\ ${ }^{d}$ Division of Operative Dentistry, College of Dental Medicine, Rangsit University, Pathum Thani, Thailand \\ ${ }^{e}$ Section of Oral Implantology, Department of Oral Rehabilitation, Fukuoka Dental College, Fukuoka, Japan
}

Keiko Nakamura ${ }^{\mathrm{a}}$, Tomohiro Kawaguchi ${ }^{\mathrm{b}}$, Hiroshi Ikeda ${ }^{\mathrm{c},}$, Pirat Karntiang $^{\mathrm{c}, \mathrm{d}}$, Kae Kakura ${ }^{\mathrm{e}}$, Yusuke Taniguchi ${ }^{\mathrm{e}}$,

\begin{abstract}
Purpose: Screw-retained implant crowns used as dental implants comprise a zirconia coping and titanium base bonded using resin cement. These devices are prone to debonding failures. This study investigated the bond characteristics of implant materials based on shear bond strength (SBS) and surface characteristics.

Methods: Chemically pure (CP) titanium grade-4 (Ti), Ti-6Al-4V alloy (Ti-6Al-4V), and tetragonal polycrystalline zirconia (zirconia) were evaluated as adherent materials. Plates of each material were polished, primed for the respective resin cements, and cemented using either methyl methacrylate-based resin cement (Super-Bond) or composite-based resin cement (Panavia). The cemented samples were subjected to 10,000 thermocycles alternating between 5 and $55{ }^{\circ} \mathrm{C}$, and the SBS were obtained before and after thermocycling. The sample surfaces were characterized based on surface observations, roughness, and free energy (SFE).

Results: The SBSs of all materials bonded using Panavia were significantly compromised during thermocycling and reached zero. Although the SBSs of Ti and Ti-6Al-4V bonded using Super-Bond were not significantly affected by thermocycling, those of zirconia decreased significantly. The bond durability between zirconia and Super-Bond was improved via alumina air-abrasion, which caused no significant loss of SBS after thermocycling. Surface analyses of the airabraded zirconia validated these results and confirmed that its surface roughness and SFE were significantly increased.

Conclusions: The bond durability between resin cement and zirconia was lower than that between Ti and Ti-6Al-4V. The alumina air-abrasion pretreatment of zirconia improved the SFE and surface roughness, thereby enhancing bond durability.
\end{abstract}

Keywords: Titanium, Zirconia, Implant, Cementing, Bond strength

Received 2 December 2020, Accepted 27 April 2021, Available online 31 August 2021

\section{Introduction}

Titanium and titanium alloys, owing to their outstanding mechanical properties and biocompatibility, have been widely used as abutment materials for dental implants. Furthermore, zirconia has been used as a coping material for abutments owing to its high mechanical strength and esthetics. This combination of titanium and zirconia has been widely used for dental implants in recent years. Furthermore, screw-retained implant crowns comprising a zirconia coping and a titanium base have exhibited exceptional fracture resistance and esthetic properties [1]. These crowns are classified into two types based on their configurations: a single unit comprising zirconia connected to the implant or a two-piece unit comprising zirconia coping and a titanium base. The titanium base in the two-piece configuration is inserted into an access hole in the zirconia

\footnotetext{
* Corresponding author at: Division of Biomaterials, Department of Oral Functions, Kyushu Dental University, Fukuoka 803-8580, Japan.

E-mail address: r16ikeda@fa.kyu-dent.ac.jp (H. Ikeda).

coping at one end and into the implant connection at the other, where the zirconia and titanium components are bonded using a luting agent (e.g., resin cement). Although the two-piece configuration offers easier handling, also it is associated with debonding failures between the zirconia crown and titanium base [2]. Several studies have attempted to overcome this problem by examining the retention strength between zirconia and titanium bonded via various bonding treatment protocols [2-7]. However, the debonding failure remains an open research problem.

The bond durability of titanium [4, 8-27] or zirconia [28-45] to resin cements has been widely investigated for restorative applications in the field of materials science. These previous studies have indicated that bond strength is enhanced by roughening the material surfaces or by priming the surface with an adhesive monomer. Zirconia and titanium can be roughened either mechanically or chemically. Mechanical roughening includes alumina air-abrasion [24, 42] and laser abrasion [27, 45], whereas chemical roughening is achieved via surface etching using hydrofluoric acid $[12,31]$ or corrodible regents $[19,25,46]$, tribochemical coating [22, $34]$, and thin-film coating [18, 32, 44].

Improvements in the bond strength can also be achieved using a 10-methacryloyloxydecyl dihydrogen phosphate (MDP)-based adhesive monomer, which is applied to both zirconia and titanium $[2,3,5,6]$. 
Several studies have reported that the combined use of alumina air-abrasion and MDP-based primers can effectively improve the bond strength between zirconia or titanium and resin cement. These studies have examined the bond strength of either the zirconia/cement or titanium/cement interface. However, no study has systematically compared the bond durability at both interfaces. Therefore, it remains unclear whether the zirconia/cement or titanium/cement interface is the weak point in the two-piece screw-retained implant crowns.

The objective of this study was to clarify the bond characteristics of zirconia and titanium to resin cement based on shear bond strength (SBS) testing and surface analyses of the materials. SBS were obtained before and after thermocycling, where two types of resin cements, namely, methyl methacrylate (MMA)-based resin cement (Super-bond) and compositebased resin cement (Panavia). Surface analyses involved scanning electron microscopy (SEM), surface roughness, and surface free energy (SFE) analyses. Alumina air-abrasion was also performed on the material surface to improve the bond strength, and the correlation between bond durability and the surface state was discussed.

\section{Materials and Methods}

\subsection{Materials}

Table 1 lists the adherent materials used in this study, including commercially pure (CP) titanium grade 4 (Ti), Ti-6Al-4V alloy (Ti$6 \mathrm{Al}-4 \mathrm{~V}$ ), and tetragonal polycrystalline zirconia (zirconia). The three materials were cut into plates $(10 \mathrm{~mm} \times 10 \mathrm{~mm} \times 1 \mathrm{~mm})$ and polished using emery paper up to \#600. The polished Ti, Ti-6Al-4V, and zirconia plates were ultrasonically cleaned using distilled water and air dried before characterization. The polished zirconia plates were subjected to alumina air-abrasion with $50-\mu \mathrm{m}$ particles using an airborne particle abrader at a pressure of $0.3 \mathrm{MPa}$ for $15 \mathrm{~s}$, which was followed by characterization.

Table 2 lists the luting materials, including Super-Bond paired with the Super-Bond PZ Primer (Sunmedical Co., Ltd), and Panavia V5 paired with the Clearfil Ceramic Primer Plus (Kuraray Noritake Dental Inc).

\subsection{Scanning electron microscopy}

The surfaces of the polished Ti, Ti-6Al-4V, zirconia, and alumina airabraded zirconia plates were analyzed using SEM (JSM-6330F; JEOL Ltd.).

\subsection{Confocal laser microscopy}

The surface roughness of the polished Ti, Ti-6Al-4V, zirconia, and alumina air-abraded zirconia plates was measured using confocal laser scanning microscopy (CLSM; VKX-100, Keyence Corp.). The mean roughness value was determined based on ten measurements for each sample.

\subsection{Surface free energy analysis}

The SFEs of the polished Ti, Ti-6Al-4V, zirconia, and alumina airabraded zirconia plates were determined by measuring the contact angle of each sample with two test liquids ( $2 \mu \mathrm{L}$ droplets), namely, distilled water $\left(\mathrm{H}_{2} \mathrm{O}\right)$ and diiodomethane $\left(\mathrm{CH}_{2} \mathrm{I}_{2}\right)$. The measurements were obtained under atmospheric conditions at room temperature $\left(20 \pm 3{ }^{\circ} \mathrm{C}\right)$ using a contact angle meter (DMe-211, Kyowa Interface Science Co., Ltd.). The mean contact angle value for each liquid was determined based on ten measurements, each acquired from a newly polished sample. The SFE of each material was calculated based on the contact angles using analytical software (FAMAS, Kyowa Interface Science Co., Ltd.) in accordance with the Owens-Wendt theory [47], as given in the following equations:
Table 1. Adherent materials used in this study.

\begin{tabular}{lll}
\hline Material & Manufacturer & Composition $(\%)$ \\
\hline CP Titanium grade 4 & NIPPON & $\mathrm{C}:<0.08, \mathrm{~N}:<0.05, \mathrm{H}: 0.013, \mathrm{Fe}: 0.5$, \\
& STEEL Corp. & $\mathrm{O}: 0.4, \mathrm{Ti}: \mathrm{Balance}$ \\
\hline Ti-6Al-4V alloy & NIPPON & $\mathrm{C}:<0.08, \mathrm{~N}:<0.05, \mathrm{H}: 0.015, \mathrm{Fe}: 0.4$, \\
& STEEL Corp. & $\mathrm{O}: 0.2, \mathrm{Al}: 6, \mathrm{~V}: 4, \mathrm{Ti}:$ Balance \\
\hline Tetragonal polycrystalline & NIPPON & $\mathrm{ZrO}_{2}: 93, \mathrm{Y}_{2} \mathrm{O}_{3}: 4.9 \%, \mathrm{HfO}_{2}: 1.8 \%$, \\
zirconia & STEEL Corp. & $\mathrm{Al}_{2} \mathrm{O}_{3}: 0.21 \%$, Other:0.3\% \\
\hline
\end{tabular}

Table 2. Luting materials used in this study.

\begin{tabular}{|c|c|c|c|}
\hline Material type & Trade name & Manufacturer & Composition \\
\hline $\begin{array}{l}\text { MMA-based resin } \\
\text { cement }\end{array}$ & Super-Bond & $\begin{array}{l}\text { Sunmedical } \\
\text { Co., Ltd }\end{array}$ & $\begin{array}{l}\text { Liquid: 4-META, MMA, } \\
\text { Powder: PMMA, TiO2 } \\
\text { Catalyst: TBB }\end{array}$ \\
\hline Primer & $\begin{array}{l}\text { Super-Bond } \\
\text { PZ Primer }\end{array}$ & $\begin{array}{l}\text { Sunmedical } \\
\text { Co., Ltd }\end{array}$ & $\begin{array}{l}\text { Liquid A: MDP, MMA } \\
\text { Liquid B: } \gamma \text {-MPTS, MMA }\end{array}$ \\
\hline $\begin{array}{l}\text { Composite-based } \\
\text { resin cement }\end{array}$ & Panavia V5 & $\begin{array}{l}\text { Kuraray } \\
\text { Noritake } \\
\text { Dental Inc. }\end{array}$ & $\begin{array}{l}\text { Paste A and B: Bis-GMA, } \\
\text { TEGDMA, Hydrophobic } \\
\text { aromatic dimethacrylate, } \\
\text { Hydrophilic aliphatic } \\
\text { dimethacrylate, Initiators, } \\
\text { Accelerators, Silanated } \\
\text { barium glass filler, Silanated } \\
\text { fluoroalminosilicate glass filler, } \\
\text { Colloidal silica Bisphenol A } \\
\text { Silanated alminium oxide filler, } \\
\text { DL-Camphorquinone, Pigments }\end{array}$ \\
\hline Primer & $\begin{array}{l}\text { Clearfil } \\
\text { Ceramic } \\
\text { primer Plus }\end{array}$ & $\begin{array}{l}\text { Kuraray } \\
\text { Noritake } \\
\text { Dental Inc. }\end{array}$ & MDP, $\gamma$-MPTS, Ethanol \\
\hline
\end{tabular}

PMMA: poly(methyl methacrylate), 4-META: 4-methacryloxyethyl trimellitate anhydride, MMA: methyl methacrylate, TBB: Tri-n-butylborane, MDP:

10-methacryloyloxydecyl dihydrogen phosphate, $\gamma$-MPTS: 3-methacryloxypropyl trimethoxy silane, Bis-GMA: bisphenol A-glycidyl methacrylate, TEGDMA: triethylene glycol dimethacrylate

$$
\begin{aligned}
& \sqrt{\gamma_{L 1}^{d} \gamma_{S}^{d}}+\sqrt{\gamma_{L 1}^{p} \gamma_{S}^{p}}=\frac{\gamma_{L 1}^{\text {total }}\left(1+\cos \theta_{L 1}\right)}{2} \\
& \sqrt{\gamma_{L 2}^{d} \gamma_{S}^{d}}+\sqrt{\gamma_{L 2}^{p} \gamma_{S}^{p}}=\frac{\gamma_{L 2}^{\text {total }}\left(1+\cos \theta_{L 2}\right)}{2} \\
& \gamma^{\text {total }}=\gamma^{d}+\gamma^{p}
\end{aligned}
$$

where subscripts $L 1$ and $L 2$ denote the test liquids $\mathrm{H}_{2} \mathrm{O}$ and $\mathrm{CH}_{2} \mathrm{I}_{2}$, respectively; $\gamma^{\text {total }}, \gamma^{\mathrm{p}}$, and $\gamma^{\mathrm{d}}$ are the total SFE, polar (hydrogen) component of the SFE, and dispersive component of the SFE for the sample material, respectively; $\gamma_{\mathrm{L}}{ }^{\text {total }}, \gamma_{\mathrm{L}}{ }^{\mathrm{p}}$, and $\gamma_{\mathrm{L}}{ }^{\mathrm{d}}$ are the total SFE, polar component of the SFE, and disperse component of the SFE of the test liquid, respectively; and $\theta$ is the contact angle for the test liquid. Previously reported SFE values were used for the test liquids [47], specifically $\gamma_{\mathrm{L} 1}{ }^{\text {total }}=72.8 \mathrm{mN} / \mathrm{m}$ $\left(\mathrm{mJ} / \mathrm{m}^{2}\right), \gamma_{\mathrm{L} 1}{ }^{\mathrm{p}}=51.0 \mathrm{mN} / \mathrm{m}$, and $\gamma_{\mathrm{L} 1}{ }^{\mathrm{d}}=21.8 \mathrm{mN} / \mathrm{m}$ for $\mathrm{H}_{2} \mathrm{O}$; and $\gamma_{\mathrm{L} 2}{ }^{\text {total }}=50.8$ $\mathrm{mN} / \mathrm{m}, \gamma_{\mathrm{L} 2}{ }^{\mathrm{p}}=1.3 \mathrm{mN} / \mathrm{m}$, and $\gamma_{\mathrm{L} 2}{ }^{\mathrm{d}}=49.5 \mathrm{mN} / \mathrm{m}$ for $\mathrm{CH}_{2} \mathrm{I}_{2}$.

\subsection{Shear bond strength test}

A polished Ti, Ti-6Al-4V, zirconia, or alumina air-abraded zirconia plate was fixed in an acrylic ring using a self-cured resin. A Teflon tube (inner diameter $=5 \mathrm{~mm}$, height $=5 \mathrm{~mm}$ ) was fixed on the sample surface to ensure a constant area for adhesion. The sample was cemented using either Super-Bond or Panavia resin cement. For the Super-Bond resin cement, the Super-Bond PZ primer was applied to the sample surface and dried under ambient conditions. Super-Bond was loaded onto the primed surface and cured under ambient conditions for $1 \mathrm{~h}$. For the Panavia resin cement, a ceramic primer was applied to the sample surface and dried under ambient conditions. Panavia was loaded onto the primed surface and light-cured for $10 \mathrm{~s}$ using a light-curing unit. After cement curing, the Teflon tube was removed from the sample surface, and the sample was held at room 
temperature for $24 \mathrm{~h}$ before being submerged in distilled water at $37{ }^{\circ} \mathrm{C}$ for $24 \mathrm{~h}$.

The SBSs of the samples $(n=12)$ were obtained before and after thermocycling, which involved alternating immersion in 5 and $55^{\circ} \mathrm{C}$ water baths for 10,000 cycles with a 60 -s dwell time for each temperature. The SBS tests were performed using a blade-shaped loading device and a mechanical testing machine (AUTOGRARH AGS-J, Shimadzu Corp.) at a crosshead speed of $1.0 \mathrm{~mm} / \mathrm{min}$. The resulting debonded surface of the sample was analyzed using an optical microscope to classify the failure mode as either adhesive failure at the cement/material interface or cohesive failure within the cement.

\subsection{Statistical analysis}

Statistical software (EZR; Saitama Medical Center, Jichi Medical University) was used to analyze the SBSs of each group using a two-way analysis of variance (ANOVA), where the type of the material (Ti, Ti$6 \mathrm{Al}-4 \mathrm{~V}$, or zirconia) and thermocycling (no or 10,000-thermocycle) were independent factors. Tukey's post-hoc test was performed when the twoway ANOVA of a group was significant. The statistical difference between the SBSs for the groups with and without alumina air-abrasion was determined using Student's t-test. The SFE and surface roughness of each group were evaluated using one-way ANOVA followed by Tukey's posthoc test. The significance level was 0.05 for all analyses.

\section{Results}

\subsection{Shear bond strength of the unabraded samples}

The two-way ANOVA of the unabraded samples indicated that the SBSs of the samples bonded with both Super-Bond and Panavia were significantly affected by the type of material $(\mathrm{p}<0.05)$ and thermocycling $(\mathrm{p}$ $<0.05$ ). Tukey's post-hoc test of the Super-Bond group indicated that the SBSs of the Ti and Ti-6Al-4V alloy did not decrease during thermocycling, whereas that of zirconia decreased significantly (Table 3 ). In contrast, the SBSs of all the materials in the Panavia group were significantly affected and decreased to zero after thermocycling.

The fracture modes between the sample materials (namely, Ti, Ti$6 \mathrm{Al}-4 \mathrm{~V}$ alloy, and zirconia) and the resin cements after SBS testing were examined and classified as adhesive and cohesive failures (Table 4). Generally, the zirconia samples cemented using Super-Bond underwent adhesive failures before and after thermocycling, whereas Ti and Ti-6Al$4 \mathrm{~V}$ alloy predominantly exhibited cohesive failure before thermocycling and adhesive failure afterwards. Before thermocycling, the Panavia samples exhibited predominantly adhesive failure, with most instances in Ti-6Al-4V, followed by zirconia and Ti. All the materials cemented using Panavia exhibited the detachment of the cement from the material surface during the thermocycling process; therefore, SBS could not be measured.

\subsection{Shear bond strength of alumina air-abraded zirconia cemented with Super-Bond}

The before- and after-thermocycling SBSs of the air-abraded zirconia cemented using Super-Bond did not differ significantly (Table 5) and all samples exhibited adhesive failure.

\subsection{Surface state of the unabraded samples}

The SEM images of the unabraded Ti, Ti-6Al-4V, and zirconia samples exhibited flat surfaces with somescratches formed during polishing (Fig. 1). The CLSM analysis indicated that the surface roughness of zirconia was significantly lower than those of Ti and Ti-6Al-4V (Table 6). These values of the surface roughness were comparable to those of the as-machined samples (Ti, Ti-6Al-4V, and zirconia) used for implant materials (data not shown here).

The dispersive component of the SFE value of unabraded zirconia was significantly lower than that of $\mathrm{Ti}$ and comparable to that of Ti-6Al-4V, whereas the polar component of zirconia was significantly lower than those
Table 3. Shear bond strengths (MPa) between unabraded materials or alumina airabraded zirconia and each cement (Super-Bond, Panavia) with no-thermocycle and 10,000-thermocycle. The different letters indicate a significant difference in each cement group $(\mathrm{p}<0.05$, Tukey test, $\mathrm{n}=12)$.

\begin{tabular}{llcc}
\hline \multirow{2}{*}{ Cement } & \multirow{2}{*}{ Material } & \multicolumn{2}{c}{ Mean value (standard deviation) } \\
\cline { 3 - 4 } Super-Bond & Ti & $16.8(2.6) \mathrm{a}$ & $17.6(3.7) \mathrm{a}$ \\
& Ti-6Al-4V & $18.9(2.3) \mathrm{a}$ & $17.9(3.0) \mathrm{a}$ \\
& Zirconia & $19.6(2.7) \mathrm{a}$ & $6.7(7.1) \mathrm{b}$ \\
\multirow{3}{*}{ Panavia } & Ti & $14.6(6.3) \mathrm{A}$ & $0(-)$ \\
& Ti-6Al-4V & $8.6(4.3) \mathrm{B}$ & $0(-)$ \\
& Zirconia & $8.0(3.6) \mathrm{B}$ & $0(-)$ \\
\hline
\end{tabular}

Table 4. Failure modes for each sample after the shear bond strength tests. Adhesive failure: Adhesive failure at the cement/material interface; Cohesive failure: cohesive failure within the cement. For the groups for Panavia with 10,000-thermocyle, the resin cements of all samples were detached from the material surface before the shear bond strength test.

\begin{tabular}{llcc}
\hline \multirow{2}{*}{ Cement } & \multirow{2}{*}{ Material } & \multicolumn{2}{c}{ Adhesive failure / Cohesive failure } \\
\cline { 3 - 4 } Super-Bond & No-thermocycle & 10,000 -thermocycle \\
\hline \multirow{6}{*}{ Panavia } & Ti & $5 / 7$ & $12 / 0$ \\
& Ti-6Al-4V & $0 / 12$ & $10 / 2$ \\
& Zirconia & $9 / 3$ & $12 / 0$ \\
& Ti & $8 / 4$ & $-/-$ \\
& Ti-6Al-4V & $12 / 0$ & $-/-$ \\
& Zirconia & $10 / 2$ & $-/-$ \\
\hline
\end{tabular}

Table 5. Mean value and standard deviation for shear bond strengths (MPa) between Super-Bond and alumina air-abraded zirconia with no-thermocycle and 10,000 -thermocycle. There is no statistical difference between the groups $(\mathrm{p}<0.05$, Student $t$ test, $\mathrm{n}=12$ )

\begin{tabular}{cc}
\hline No-thermocycle & 10,000 -thermocycle \\
\hline $22.0(2.5)$ & $21.5(2.5)$ \\
\hline
\end{tabular}
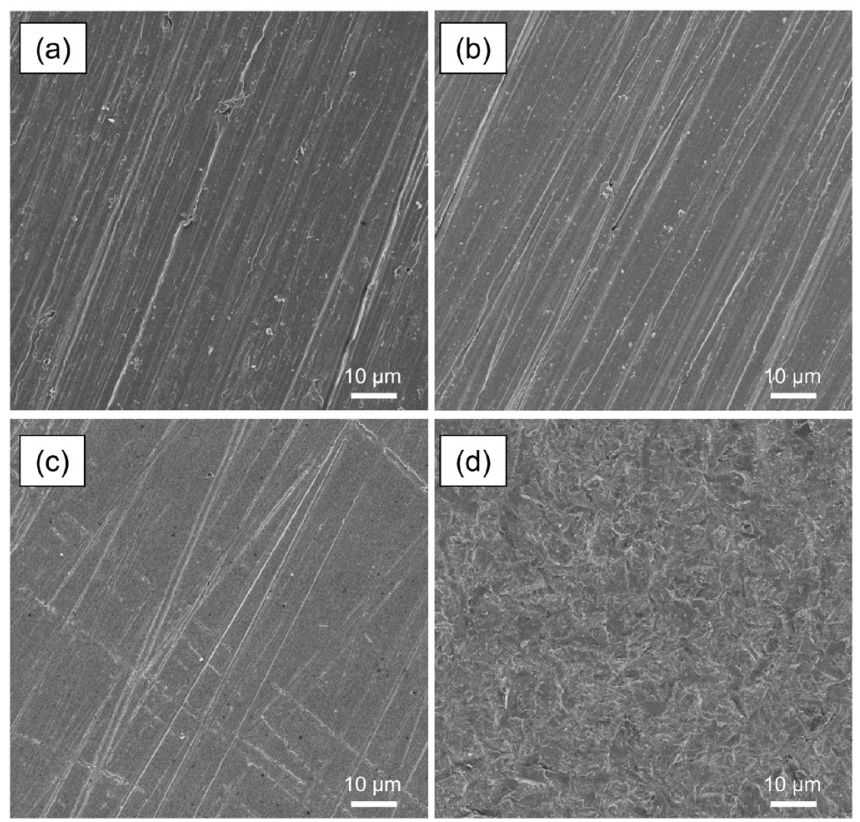

Fig. 1. SEM images of unabraded (a) Ti, (b) Ti-6Al-4V, (c) zirconia, and (d) alumina air-abraded zirconia.

of Ti and 6Ti-6Al-4V alloy (Table 7). Therefore, the total SFE of zirconia was significantly lower than those of Ti and Ti-6Al-4V. 
Table 6. Mean value and standard deviation for surface roughness ( $\mathrm{Ra}, \mu \mathrm{m})$ of unabraded Ti, Ti-6Al-4V, zirconia, and alumina air-abraded zirconia, measured by the confocal laser scanning microscope. The different letters indicate a significant difference in the groups $(\mathrm{p}<0.05$, Tukey test, $\mathrm{n}=12)$.

\begin{tabular}{cccc}
\hline $\mathrm{Ti}$ & Ti-6Al-4V & Zirconia & Abraded zirconia \\
\hline $0.26(0.09) \mathrm{a}$ & $0.25(0.05) \mathrm{a}$ & $0.11(0.02) \mathrm{b}$ & $0.34(0.06) \mathrm{c}$ \\
\hline
\end{tabular}

Table 7. Mean value and standard deviation for surface free energy $(\mathrm{mN} / \mathrm{m})$ of unabraded Ti, Ti-6Al-4V, zirconia, and alumina air-abraded zirconia. The different letters indicate a significant difference in the groups for each component $(\mathrm{p}<0.05$, Tukey test, $\mathrm{n}=10$ ).

\begin{tabular}{lccc}
\hline Material & Dispersive component & Polar component & Total \\
\hline Ti & $36.6(0.6) \mathrm{a}$ & $36.8(0.9) \mathrm{A}$ & $73.4(1.0) \alpha$ \\
Ti-6Al-4V & $34.0(1.4) \mathrm{b}$ & $38.6(1.2) \mathrm{A}$ & $72.5(0.6) \alpha$ \\
Zirconia & $33.0(1.2) \mathrm{bc}$ & $26.3(2.6) \mathrm{B}$ & $59.3(2.7) \beta$ \\
Abraded zirconia & $32.6(0.4) \mathrm{c}$ & $37.6(1.1) \mathrm{A}$ & $70.2(1.0) \chi$ \\
\hline
\end{tabular}

\subsection{Surface state of alumina air-abraded zirconia}

The SEM image (Fig. 1(d)) and the three-dimensional (3D) CLSM image (Fig. 2) of the alumina air-abraded zirconia surface depict that the sample surface was roughened by alumina air-abrasion. Alumina airabrasion significantly increased the surface roughness of zirconia compared to that of the unabraded samples (Table 6).

The polar component and total SFE of zirconia were significantly larger after abrasion, whereas its dispersive component remained almost unchanged (Table 7).

\section{Discussion}

The SBS measurements demonstrated that the bond durability offered by Super-Bond was superior to that of Panavia (Table 3). This result was supported by the evaluation of fracture modes (Table 4). This finding corresponds to a previous report on the retentive force of zirconia copings bonded to zirconia abutments using resin cements. In that study, SuperBond exhibited a higher retentive force when compared with that of Panavia [48]. Super-Bond contains no filler, which may have enabled better flow on the material surfaces to enhance the bond strength.

The SBS results also indicated that the bond durability of Super-Bond to zirconia was lower than those of Ti and Ti-6Al-4V. Therefore, a twopiece zirconia implant crown with a titanium base and zirconia coping bonded using Super-Bond is expected to fail first at the zirconia/resin cement interface that at the titanium/resin cement interface. The lower bond durability of zirconia than that of titanium was further investigated based on the surface roughness and SFE analysis. The surface roughness of zirconia was significantly lower than those of Ti and Ti-6Al-4V (Table 6). This was probably owing to the higher hardness of zirconia when compared with that of titanium. This lower surface roughness of zirconia contributed to the lower bond durability using the resin cement. The chemical state of the zirconia surface was also a factor, and the SFE analysis indicated that the polar component of zirconia was significantly lower than those of $\mathrm{Ti}$ and Ti-6Al-4V. Therefore, the zirconia surface had fewer active sites to react with the adhesive MDP monomer when compared with those of $\mathrm{Ti}$ or Ti-6Al-4V. Therefore, the hydrolytic degradation at the zirconia/resin cement interface progressed more rapidly.

It was inferred from the results listed in Table 3 that while there is scope to improve the bond durability of zirconia using Super-bond. Therefore, alumina air-abrasion was performed on zirconia for the Super-Bond group. The bond durability between zirconia and the Super-Bond resin cement was improved by alumina air-abrasion, whereas the enhanced bond durability and SBS were not significantly changed by thermocycling (Table 5). Previous studies on the improvement of bond strength via alumina airabrasion have reported similar findings [24, 42, 49-53]. The mechanism of bond durability improvement by alumina air-abrasion was investigated based on surface analysis. The surface roughness of zirconia increased after alumina air-abrasion, which enhanced the mechanical interrocking between
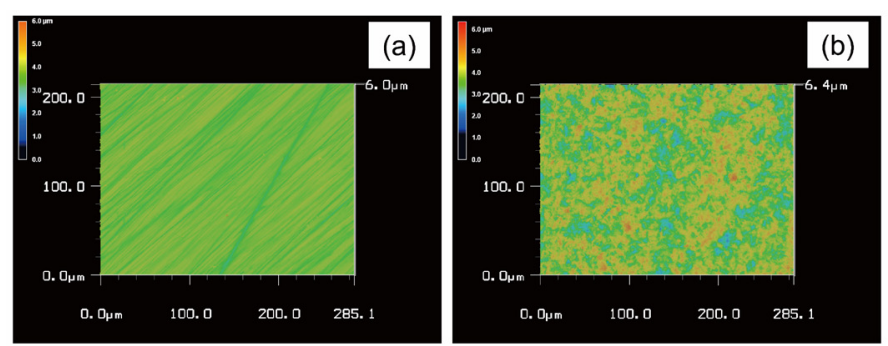

Fig. 2. Three-dimensional image of the zirconia surface measured by the confocal laser scanning microscope, (a) unabraded zirconia, (b) the alumina air-abraded zirconia surface. The surface roughness $(\mathrm{Ra})$ of the no-abraded zirconia and the alumina air-abraded zirconia was $0.11 \pm 0.02 \mu \mathrm{m}$ and $0.34 \pm 0.06 \mu \mathrm{m}$, respectively.

the zirconia surface and resin cement to improve the bond strength [37]. Furthermore, the SFE analysis indicated that alumina air-abrasion caused an increase in the polar component, thereby increasing the total SFE of zirconia samples. The SFE of a material is typically affected by the number of functional groups (e.g., OH groups) on its surface [54]. It was speculated that the increased SFE and wettability after alumina air-abrasion were attributed to an increased number of surface $\mathrm{OH}$ groups or other functional groups on zirconia. Studies on the chemical interaction between the MDP monomer and zirconia [55] indicate that the MDP monomer can be adsorbed onto zirconia via hydrogen bonding between the $\mathrm{P}=\mathrm{O}$ of the MDP monomer and the surface $\mathrm{OH}$ groups of zirconia or via ionic interaction between $\mathrm{P}-\mathrm{O}-$ and $\mathrm{Zr}+$. This mechanism demonstrates that the surface $\mathrm{OH}$ groups of zirconia serve as active sites for the bonding of the resin cement via the MDP monomer. Therefore, an increase in the number of surface $\mathrm{OH}$ groups after alumina air-abrasion improves the bond strength of zirconia samples. Although several studies have evaluated the zirconia surface based on the SFE analysis [56-61], the changes in SFE caused by alumina air-abrasion have not yet been investigated. This is the first demonstration of alumina air-abrasion increasing the surface roughness and SFE, both of which contributed to the improved bond durability between zirconia and resin cement.

Within the limitations of this study, MMA-based resin cement (SuperBond) is recommended for the bonding of zirconia and titanium (or titanium alloy) in two-piece zirconia implant crowns. Furthermore, alumina air-abrasion is an effective pretreatment of the zirconia surface to improve the bond durability between the resin and zirconia, which is the interface more vulnerable to failure. To improve the retention of zirconia coping to the titanium base, previous studies recommended the use of an MDPbased primer, followed by alumina air-abrasion before cementing [2, 3]. In addition to this pretreatment, the present study suggests that the MMAbased resin cement is effective for zirconia and titanium. The alumina airabraded zirconia combined with the MMA-based resin cement exhibited almost no deterioration in bond strength during thermocycling. Therefore, it may be possible to reduce the debonding failure of zirconia copings and their titanium bases in two-piece implant crowns. Further in vitro investigations are necessary to confirm the validity of these findings under clinical situations.

\section{Conclusion}

This study demonstrated that MMA-based resin cement offered superior bonding between titanium (CP titanium grade- 4 or Ti-6Al-4V alloy) and zirconia than that offered by composite-based resin cements. The bond durability between resin cement and titanium was higher than that using zirconia because the surface roughness and free energy of titanium were higher. The pretreatment of zirconia via alumina air-abrasion enhanced its surface roughness and free energy, thereby improving the bond durability between zirconia and the MMA-based resin cement.

\section{Conflict of Interest}

The authors declare that there is no conflict of interest. 


\section{References}

[1] Sailer I, Muhlemann S, Zwahlen M, Hammerle CH, Schneider D. Cemented and screw-retained implant reconstructions: a systematic review of the survival and complication rates. Clin Oral Implants Res. 2012;23:163-201.

[2] von Maltzahn NF, Holstermann J, Kohorst P. Retention forces between titanium and zirconia components of two-part implant abutments with different techniques of surface modification. Clin Implant Dent Relat Res. 2016;18:73544

[3] Gehrke P, Alius J, Fischer C, Erdelt KJ, Beuer F. Retentive strength of twopiece CAD/CAM zirconia implant abutments. Clin Implant Dent Relat Res. 2014;16:920-5.

[4] Arce C, Lawson NC, Liu PR, Lin CP, Givan DA. Retentive force of zirconia implant crowns on titanium bases following different surface treatments. Int J Oral Maxillofac Implants. 2018;33:530-5.

[5] Freifrau von Maltzahn N, Bernard S, Kohorst P. Two-part implant abutments with titanium and ceramic components: Surface modification affects retention forces-An in-vitro study. Clin Oral Implants Res. 2019;30:903-9.

[6] Hansen NA, Wille S, Kern M. Effect of reduced airborne particle abrasion pressure on the retention of zirconia copings resin bonded to titanium abutments. J Prosthet Dent. 2019;124:60-7.

[7] Linkevicius T, Caplikas A, Dumbryte I, Linkeviciene L, Svediene O. Retention of zirconia copings over smooth and airborne-particle-abraded titanium bases with different resin cements. J Prosthet Dent. 2019;121:949-54.

[8] Taira Y, Matsumura H, Yoshida K, Tanaka T, Atsuta M. Influence of surface oxidation of titanium on adhesion. J Dent. 1998;26:69-73

[9] Taira Y, Yanagida H, Matsumura H, Yoshida K, Atsuta M, Suzuki S. Adhesive bonding of titanium with a thione-phosphate dual functional primer and selfcuring luting agents. Eur J Oral Sci. 2000;108:456-60.

[10] Schneider R, de Goes MF, Henriques GE, Chan DC. Tensile bond strength of dual curing resin-based cements to commercially pure titanium. Dent Mater. 2007;23:81-7.

[11] Kurt M, Kulunk T, Ural C, Kulunk S, Danisman S, Savas S. The effect of different surface treatments on cement-retained implant-supported restorations. J Oral Implantol. 2013;39:44-51.

[12] Elsaka SE, Swain MV. Effect of surface treatments on the adhesion of selfadhesive resin cements to titanium. J Adhes Dent. 2013;15:65-71.

[13] Gultekin P, Gultekin BA, Aydin M, Yalcin S. Cement selection for implantsupported crowns fabricated with different luting space settings. J Prosthodont. 2013;22:112-9.

[14] Almilhatti HJ, Neppelenbroek KH, Vergani CE, Machado AL, Pavarina AC, Giampaolo ET. Adhesive bonding of resin composite to various titanium surfaces using different metal conditioners and a surface modification system. J Appl Oral Sci. 2013;21:590-6.

[15] Mehl C, Harder S, Steiner M, Vollrath O, Kern M. Influence of cement film thickness on the retention of implant-retained crowns. J Prosthodont. 2013;22:618-25

[16] Wadhwani C, Chung KH. Bond strength and interactions of machined titaniumbased alloy with dental cements. J Prosthet Dent. 2015;114:660-5.

[17] Koizumi H, Furuchi M, Tanoue N, Yanagida H, Yoneyama T, Matsumura H. Bond strength to primed Ti-6Al-7Nb alloy of two acrylic resin adhesives. Dent Mater J. 2006:25:286-90.

[18] Matinlinna JP, Lassila LV, Vallittu PK. The effect of a novel silane blend system on resin bond strength to silica-coated Ti substrate. J Dent. 2006;34:436-43.

[19] Tsuchimoto Y, Yoshida Y, Takeuchi M, Mine A, Yatani H, Tagawa Y, et al Effect of surface pre-treatment on durability of resin-based cements bonded to titanium. Dent Mater. 2006;22:545-52

[20] Fonseca RG, de Almeida JG, Haneda IG, Adabo GL. Effect of metal primers on bond strength of resin cements to base metals. J Prosthet Dent. 2009;101:262-8.

[21] Fernandes CA, Ribeiro JC, Larson BS, Bonfante EA, Silva NR, Suzuki M, et al. Microtensile bond strength of resin-based composites to Ti-6Al-4V. Dent Mater. 2009;25:655-61

[22] Abi-Rached FdO, Fonseca RG, Haneda IG, de Almeida-Júnior AA, Adabo GL. The effect of different surface treatments on the shear bond strength of luting cements to titanium. J Prosthet Dent. 2012;108:370-6.

[23] Koizumi H, Naito K, Ishii T, Yamashita M, Yoneyama T, Matsumura H. Adhesive bonding of Ti-6Al-7Nb alloy and component metals with acidic primers and a tri-n-butylborane initiated resin. J Adhes Dent. 2012;14:283-92.

[24] Fonseca RG, Haneda IG, Almeida-Junior AA, de Oliveira Abi-Rached F, Adabo GL. Efficacy of air-abrasion technique and additional surface treatment at titanium/resin cement interface. J Adhes Dent. 2012;14:453-9.

[25] Elsaka SE. Effectiveness of titanium tetrafluoride on the bond strength of composite cement to titanium. J Adhes Dent. 2018;20:143-9.

[26] Cao Y, Guo YY, Chen L, Han J, Tong H, Zhang B, et al. Effects of different surface treatments on bond strength of resin cement to machined pure titanium. J Adhes Dent. 2019;21:401-11.

[27] Ates SM, Korkmaz FM, Caglar IS, Duymus ZY, Turgut S, Bagis EA. The effect of ultrafast fiber laser application on the bond strength of resin cement to titanium. Lasers Med Sci. 2017;32:1121-9.

[28] Kern M, Wegner SM. Bonding to zirconia ceramic: adhesion methods and their durability. Dent Mater. 1998;14:64-71

[29] Lee S-E, Bae J-H, Choi J-W, Jeon Y-C, Jeong C-M, Yoon M-J, et al. Comparative shear-bond strength of six dental self-adhesive resin cements to zirconia. Materials. 2015;8:3306-15.

[30] Zhao L, Jian YT, Wang XD, Zhao K. Bond strength of primer/cement systems to zirconia subjected to artificial aging. J Prosthet Dent. 2016;116:790-6.
[31] Lee JH, Lee CH. Effect of the surface treatment method using airborne-particle abrasion and hydrofluoric acid on the shear bond strength of resin cement to zirconia. Dent J. 2017;5:23

[32] Thammajaruk P, Buranadham S, Thanatvarakorn O, Guazzato M. Influence of nano-structured alumina coating on composite-zirconia bonding and its characterization. J Adhes Dent. 2018;20:233-42.

[33] Yagawa S, Komine F, Fushiki R, Kubochi K, Kimura F, Matsumura H. Effect of priming agents on shear bond strengths of resin-based luting agents to a translucent zirconia material. J Prosthodont Res. 2018;62:204-9.

[34] Akazawa N, Koizumi H, Nogawa H, Nakayama D, Kodaira A, Matsumura H. Effect of mechanochemical surface preparation on bonding to zirconia of a trin-butylborane initiated resin. Dent Mater J. 2017;36:19-26.

[35] Miyazaki T, Nakamura T, Matsumura H, Ban S, Kobayashi T. Current status of zirconia restoration. J Prosthodont Res. 2013;57:236-61.

[36] Inokoshi M, De Munck J, Minakuchi S, Van Meerbeek B. Meta-analysis of bonding effectiveness to zirconia ceramics. J Dent Res. 2014;93:329-34.

[37] Ozcan M, Bernasconi M. Adhesion to zirconia used for dental restorations: a systematic review and meta-analysis. J Adhes Dent. 2015;17:7-26.

[38] Tzanakakis EG, Tzoutzas IG, Koidis PT. Is there a potential for durable adhesion to zirconia restorations? A systematic review. J Prosthet Dent. 2016;115:9-19.

[39] Khan AA, Al Kheraif AA, Jamaluddin S, Elsharawy M, Divakar DD. Recent trends in surface treatment methods for bonding composite cement to zirconia: A reveiw. J Adhes Dent. 2017;19:7-19.

[40] Scaminaci Russo D, Cinelli F, Sarti C, Giachetti L. Adhesion to zirconia: A systematic review of current conditioning methods and bonding materials. Dent J. 2019;7:74.

[41] Quigley NP, Loo DSS, Choy C, Ha WN. Clinical efficacy of methods for bonding to zirconia: A systematic review. J Prosthet Dent. 2020;In press.

[42] Abi-Rached FO, Martins SB, Almeida-Junior AA, Adabo GL, Goes MS, Fonseca RG. Air abrasion before and/or after zirconia sintering: surface characterization, flexural strength, and resin cement bond strength. Oper Dent. 2015;40:E66-75

[43] Thammajaruk P, Inokoshi M, Chong S, Guazzato M. Bonding of composite cements to zirconia: A systematic review and meta-analysis of in vitro studies. J Mech Behav Biomed Mater. 2018;80:258-68

[44] Thammajaruk P, Blatz MB, Buranadham S, Guazzato M, Wang Y. Shear bond strength of composite cement to alumina-coated versus tribochemical silicatreated zirconia. J Mech Behav Biomed Mater. 2020;105:103710.

[45] Abu Ruja M, De Souza GM, Finer Y. Ultrashort-pulse laser as a surface treatment for bonding between zirconia and resin cement. Dent Mater. 2019;35:1545-56.

[46] Barutcigil K, Barutcigil C, Kul E, Ozarslan MM, Buyukkaplan US. Effect of different surface treatments on bond strength of resin cement to a CAD/CAM restorative material. J Prosthodont. 2019;28:71-8.

[47] Owens DK, Wendt DT. Estimation of the surface free energy of polymers. J Appl Polym Sci. 1969;13:1741-7.

[48] Kim SM, Yoon JY, Lee MH, Oh NS. The effect of resin cements and primer on retentive force of zirconia copings bonded to zirconia abutments with insufficient retention. J Adv Prosthodont. 2013;5:198-203.

[49] Lv P, Yang X, Jiang T. Influence of hot-etching surface treatment on zirconia/ resin shear bond strength. Materials. 2015;8:8087-96.

[50] Erdem A, Akar GC, Erdem A, Kose T. Effects of different surface treatments on bond strength between resin cements and zirconia ceramics. Oper Dent. 2014;39:E118-27.

[51] Moon JE, Kim SH, Lee JB, Ha SR, Choi YS. The effect of preparation order on the crystal structure of yttria-stabilized tetragonal zirconia polycrystal and the shear bond strength of dental resin cements. Dent Mater. 2011;27:651-63.

[52] Aung S, Takagaki T, Lyann SK, Ikeda M, Inokoshi M, Sadr A, et al. Effects of alumina-blasting pressure on the bonding to super/ultra-translucent zirconia. Dent Mater. 2019;35:730-9.

[53] Okutan Y, Yucel MT, Gezer T, Donmez MB. Effect of airborne particle abrasion and sintering order on the surface roughness and shear bond strength between Y-TZP ceramic and resin cement. Dent Mater J. 2019;38:241-9.

[54] Hołysz L. The effect of thermal treatment of silica gel on its surface free energy components. Colloids Surf, A. 1998;134:321-9.

[55] Nagaoka N, Yoshihara K, Feitosa VP, Tamada Y, Irie M, Yoshida Y, et al. Chemical interaction mechanism of 10-MDP with zirconia. Sci Rep. 2017;7:45563

[56] Kim MJ, Kim YK, Kim KH, Kwon TY. Shear bond strengths of various luting cements to zirconia ceramic: surface chemical aspects. J Dent. 2011;39:795803 .

[57] Vechiato-Filho AJ, Matos AO, Landers R, Goiato MC, Rangel EC, De Souza GM, et al. Surface analysis and shear bond strength of zirconia on resin cements after non-thermal plasma treatment and/or primer application for metallic alloys. Mater Sci Eng C Mater Biol Appl. 2017;72:284-92.

[58] Bitencourt SB, Dos Santos DM, da Silva EVF, Barao VAR, Rangel EC, da Cruz $\mathrm{NC}$, et al. Characterisation of a new plasma-enhanced film to improve shear bond strength between zirconia and veneering ceramic. Mater Sci Eng C Mater Biol Appl. 2018;92:196-205.

[59] Akazawa N, Koizumi H, Nogawa H, Kodaira A, Burrow MF, Matsumura H. Effect of etching with potassium hydrogen difluoride and ammonium hydrogen difluoride on bonding of a tri-n-butylborane initiated resin to zirconia. Dent Mater J. 2019;38:540-6.

[60] Lima RBW, Barreto SC, Alfrisany NM, Porto TS, De Souza GM, De Goes MF. Effect of silane and MDP-based primers on physico-chemical properties of zirconia and its bond strength to resin cement. Dent Mater. 2019;35:1557-67. 
[61] Lumkemann N, Eichberger M, Stawarczyk B. Different surface modifications combined with universal adhesives: the impact on the bonding properties of zirconia to composite resin cement. Clin Oral Investig. 2019;23:3941-50.

\section{(c) (i)}

Copyright: This is an open-access article distributed under the terms of Creative Commons Attribution License 4.0 (CCBY 4.0), which allows users to distribute and copy the material in any format so long as attribution is given to the author(s). 